\title{
Risk Assessment of Mentally Ill Patients are Urgently Needed in China
}

\author{
Xiao Min Zhu and Xiao Ping Wang* \\ Mental Health Institute, The Second Xiangya Hospital, Central South University, Changsha, Hunan Province, China
}

The violence risk of mentally ill patients has always been the major task and challenge of psychiatric research, and one of the focuses of public health care. According to data released by the Disease Prevention and Control Center in Chinese Health Ministry, there are more than 17 million all kinds of patients with mental disorder in China. Among them, over 1.2 million are diagnosed severe mental disorders such as schizophrenia with index offence. In recent years criminal cases are more than 1,0000/year on the implementation of mentally ill patients, $30 \%$ of them are murder or attempted murder, etc. [1,2]. Xiaoping Wang, etc. found that approximately $82.5 \%$ of these individuals had a psychiatric history prior to the index offence and $68.6 \%$ for the index offense was murder or attempted murder compared with only $10.5 \%$ of the Canadian cohort in a study [3].

Despite lack of large-scale epidemiological investigation accurate information about violent crimes of mentally ill patients is unclear; the findings suggest that the risks of violent behaviors in mental illness patients are higher than those of ordinary normal population. The violent incidents caused by mental patients hit close to $10 \%$ of the total behaviors. A large-scale epidemiological study in various countries found that violence risk was approximately $10-15 \%$ from patients diagnosed with severe mental disorders [4].

Those violent events bring increasing adverse influence such as stigma and awareness of the elevated risk of perpetrating violence in individuals with mental disorders. Chinese government also carried out profound laws and regulations to cope with the appeal. On Jan $1^{\text {st }}, 2013$, China amended Criminal Procedure Law was implemented. The section 284 stipulates: "The mentally ill offenders without criminal responsibility on account of mental disorders by legal procedures assessment, have to receive compulsory medical treatment if they are presumed to be risk of violence". On May $1^{\text {st }}, 2013$, China Mental Health Act was implemented. The second clause of Article 30 of the Mental Health Act states: "The patients with severe mental disorders should receive involuntary hospitalization under one of the following circumstances: (a) danger behavior/s or risk to self; (b) danger behavior/s or risk to others". Whether compulsory medical treatment mentioned by China amended Criminal Procedure Law or involuntary hospitalization involved in China Mental Health Act, there is a core technical problem related to implementing process of these laws, that's, risk assessment.

It is one of the main tasks of forensic psychiatry to reduce risk behaviors in mentally ill patients through risk assessment and management. Because of the subjectivity of mental disorders diagnosis and the complexity of disease itself, how to assess dangerous behavior or risk in patients with mental disorder is critically important. Relevant successful experience shows that the risk of violence in mentally ill patients can be predicted relatively accurately and controlled to some extent. Violence risk assessment has been put into mental disorders treatment guidelines by American Psychiatric Association and the British National Institute for Health and Clinical optimization [5,6]. Structured risk assessment tools such as HCR-20 have been regularly used for assisting in violence risk assessment of patients (and offenders) in some developed countries, and are often used to justify the long periods of detention [7-9]. Many patients are discharged if they at low level of risks, but much of these instruments are based on static or unchangeable risk factors, which mean that their level of risk will not materially change with treatment. Nevertheless, these tools provide some means to assist forensic psychiatrists to make these difficult decisions regarding detention and discharge.

The Chinese violence risk assessment is still in its infancy and lack of systematic research and evidence based risk assessment tools for mentally ill patients. Bin Xie, etc. introduced MOAS (Modified Overt Aggression Scale) and conducted the rater consistency to the Chinese version of MOAS [10]. My research team brought in HCR-20 (The Historical Clinical Risk Management) and carried out a preliminary study of the reliability and validity about it [11]. Xueli Zhang, et al. revised violence Risk Scale (VRS) for assessing violence risk and risk change, and examined the reliability of this modified Violence Risk Scale in Chinese version (VRS-C) [12]. However, because such assessment tools need specialized training and take long time to finish the evaluation, they are still in the research stage. No debate on the actuarial vs. structured professional judgment has been found so far. The Disease Prevention and Control Center in Chinese Health Ministry issued a "Severe mental disorders management norms" in 2009 and classified the violence risk of severe mental disorders into 6 levels from light to heavy. But no validity and reliability data was provided in Chinese patients. Therefore, it is an urgent task currently to establish a relatively objective, quantifiable, operational assessment model or tool based on evidence. The model of the tool can be used for accurate assessment and prediction the risk of mentally ill patients.

The Chinese forensic psychiatry experts mostly adopt unstructured clinical assessment to estimate violent risk of mentally ill patients based on investigation, history taking and clinical examination. This method advantage is to make individual assessment and the disadvantage is not very objective and less systematic. Different institution even different experts in the same institution may obtain different conclusions by the unstructured clinical assessment, which means the psychiatrists make the judgments based on their own clinical experience and makes it difficult to compare the assessment opinions. A few scholars specializing in forensic psychiatry or psychologists advocate the actuarial assessment or structured professional judgment. Therefore, it is necessary to develop a shared risk assessment model or tool for Chinese forensic psychiatry experts to reach a coincident opinion in one case as much as possible. But there are enormous complicated variables involved in risk assessment such as biological and psychological characteristics, environmental influence from family and society, types

*Corresponding author: Xiao Ping Wang, Mental Health Institute, The Second Xiangya Hospital, Central South University, Changsha, Hunan province, China Tel: +86-731-85292182; Fax: +86-731-85360162; E-mail: xiaop6@126.com

Received March 11, 2014; Accepted June 16, 2014; Published June 23, 2014

Citation: Zhu XM, Wang XP (2014) Risk Assessment of Mentally III Patients are Urgently Needed in China. J Forensic Res 5: 245. doi:10.4172/2157-7145.1000245

Copyright: ( 2014 Zhu XM, et al. This is an open-access article distributed under the terms of the Creative Commons Attribution License, which permits unrestricted use, distribution, and reproduction in any medium, provided the original author and source are credited. 
Citation: Zhu XM, Wang XP (2014) Risk Assessment of Mentally III Patients are Urgently Needed in China. J Forensic Res 5: 245. doi:10.4172/21577145.1000245

Page 2 of 2

of violence, incentives, illness situation, etc. [13]. It is very difficult task to design a shared assessment model or tool with considering so many factors in so urgent time to promote better implementation of China Mental Health Act and the amended Criminal Procedure Law. While it is a reasonable way to develop a risk assessment checklist, particularly focusing on modifiable risk factors based on previous research. Katrina Witt, etc. found that those four factors had a high correlation with violence risk including the conduct of hostilities, the recent substance abuse and alcohol abuse history, treatment non adherence, history of crime and violence. And they also found that the four factors could predict violence risk through a systematic literature review of 110 studies and meta-analysis [14]. At the same time, set up a database for severe mental illness patients in China and then revise the shared risk assessment checklist. Eventually China will develop a management protocol for mental illness patients based on their different level of risk and will have a profound risk assessment tool or model to promote reasonable and effective disposal, prevention and surveillance services for the mentally ill patients.

\section{References}

1. Chinese Government Document (2002-2010) Plan for china's mental health work.

2. Phillips MR, Zhang J, Shi Q, Song Z, Ding Z, et al. (2009) Prevalence, treatment, and associated disability of mental disorders in four provinces in China during 2001-05: An epidemiological survey. Lancet 373: 2041-2053.

3. Wang X, Livingston JD, Brink J, Murphy E (2006) Persons found 'not criminally responsible on account of mental disorder': a comparison of British Columbia, Canada and Hunan, China. Forensic Sci Int 164: 93-97.
4. Fazel S, Långström N, Hjern A, Grann M, Lichtenstein P (2009) Schizophrenia, substance abuse, and violent crime. JAMA 301: 2016-2023.

5. American Psychiatric Association (2004) Practice guidelines for the treatment of patients with schizophrenia.

6. London: National Institute for Health and Clinical Excellence (2009) Core interventions in the treatment and management of schizophrenia in primary and secondary care. National Institute for Health and Clinical Excellence.

7. Douglas KS, Ogloff JR, Hart SD (2003) Evaluation of a model of violence risk assessment among forensic psychiatric patients. Psychiatr Serv 54: 13721379.

8. Yang M, Wong SC, Coid J (2010) The efficacy of violence prediction: a metaanalytic comparison of nine risk assessment tools. Psychol Bull 136: 740-767.

9. McDermott BE, Edens JF, Quanbeck CD, Busse D, Scott CL (2008) Examining the role of static and dynamic risk factors in the prediction of inpatient violence: variable- and person-focused analyses. Law Hum Behav 32: 325-338.

10. Xie B, Zheng PZ (2001) Revision Modified version of Overt Aggression Scale (MOAS). Chinese Journal of Behavioral Medical Science10 (Suppl): 195-196.

11. Xiao Q, Li C, Wang XP, Jiang SA (2010) Reliability and validity study of HCR20 for violence risk assessment in schizophrenia. Journal of Clinical Research 27: 405-408

12. Zhang XL, Chen XC, Cai WX, Hu JM (2012) Reliability of the Violence Risk Scale of Chinese version. Fa Yi Xue Za Zhi 28: 32-35.

13. Singh JP, Fazel S (2010) Forensic risk assessment: A meta review. Crim Justice Behav 37: 965-988.

14. Witt K, van Dorn R, Fazel S (2013) Risk Factors for Violence in Psychosis: Systematic Review and Meta-Regression Analysis of 110 Studies. PLoS One 8: e55942. 